\title{
Electronic and optical properties of InGaN quantum dot based light emitters for solid state lighting
}

\author{
Yuh-Renn Wu, ${ }^{1, a)}$ Yih-Yin Lin, ${ }^{2}$ Hung-Hsun Huang, ${ }^{1}$ and Jasprit Singh ${ }^{2}$ \\ ${ }^{1}$ Institute of Photonics and Optoelectronics and Department of Electrical Engineering, National Taiwan \\ University, Taipei 10617, Taiwan \\ ${ }^{2}$ Department of Electrical Engineering and Computer Science, University of Michigan, Ann Arbor, Michigan \\ 48109, USA
}

(Received 3 October 2008; accepted 23 November 2008; published online 15 January 2009)

\begin{abstract}
In this paper, we have made a systematic study of the electronic and optical properties of InGaN based quantum dot light emitters. The valence force field model and $6 \times 6 k \cdot p$ method have been applied to study the band structures in InGaN or InN quantum dot devices. Piezoelectric and spontaneous polarization effects are included. A comparison with InGaN quantum wells shows that InGaN quantum dots can provide better electron-hole overlap and reduce radiative lifetime. We also find that variation in dot sizes can lead to emission spectrum that can cover the whole visible light range. For high carrier density injection conditions, a self-consistent method for solving quantum dot devices is applied for better estimation of device performance. Consequences of variations in dot sizes, shapes, and composition have been studied in this paper. The results suggest that InGaN quantum dots would have superior performance in white light emitters. (C) 2009 American Institute of Physics. [DOI: 10.1063/1.3065274]
\end{abstract}

\section{INTRODUCTION}

Nitride based light emitting diodes (LEDs) have become important ultraviolet and blue light sources. ${ }^{1,2}$ With the assistance of phosphors, these devices can provide white light emission and are very important technologies for the lighting markets. It is known that the bandgap of InGaN alloys can range from 0.7 to $3.4 \mathrm{eV}$, thus covering the emission spectrum from infrared to ultraviolet. Therefore, it is possible to make InGaN alloys as red, green, and blue (RGB) lighting sources to emit white light without the assistance of phosphors. There is also a strong potential in smart lighting markets by changing RGB colors strength. However, the emission efficiency of InGaN quantum well drops significantly when we push to longer wavelength, i.e., green or red light emission. ${ }^{3,4}$ There is a large lattice mismatch of $\sim 10 \%$ between the InN and GaN. This large lattice mismatch leads to a strong piezoelectric polarization field inside the quantum wells for the normally used $c$-axis growth. As a result, a strong quantum confined Stark effect (QCSE) is observed in the quantum well structures. The crystal film quality also worsens due to stronger strain as we push the emission to longer wavelength by increasing the indium composition. This may also lead to In-clustering effects. Therefore, it is hard to grow a high indium composition layer for the longer wavelength emission and the efficiency drops significantly for longer wavelength LEDs.

It is known that under high strain conditions StranskiKrastanov growth mode can dominate leading to dot formation. ${ }^{5-10}$ Also increased indium composition might lead to phase segregation or form a quantum-dot-like structure due to the strong lattice mismatch. In such structures the electron and hole states become localized. These localized

${ }^{a)}$ Electronic mail: yrwu@cc.ee.ntu.edu.tw. states might be disadvantages for applications that require precise control of emission spectrum such as lasers, etc. However, for lighting technologies where a broad band of emission spectrum is needed, these structures might have some advantages. As we know, the formation of quantum dot structures comes with the strain relaxation of InGaN layer so that the piezoelectric polarization effect will be weaker than the quantum well structures. Furthermore, it is much easier to grow higher indium composition of quantum dots and even $100 \%$ InN quantum dot structures can be grown. ${ }^{11}$ Therefore, by controlling the dot size and indium composition, it is possible to emit in the entire visible light emission spectrum. Hence, it is important to understand if the use of quantum dot systems has any advantage over quantum well systems in terms of e-h overlap, polarization dependence of light emission and bandgap ranges possible.

There have been some works for studying of $\mathrm{AlGaN} /$ GaN based quantum dots ${ }^{12-16}$ and some works for InGaN based quantum $\operatorname{dots}^{9}$ based on different methods to calculate the strain and electronic states, which have motivated the experimentalists to work on this field. In this work, we applied the valence force field (VFF) model ${ }^{17,18}$ to study the strain, which is a microscopic atomic model and is used to study the strain of other material systems in our laboratory. ${ }^{19,20}$ Compared to the macroscopic model such as using continuum mechanical method ${ }^{9}$ or Green's function for the strain tensor, ${ }^{14}$ VFF model provides a atomistic approach to the strain calculation. The spontaneous polarization, piezoelectric polarization induced by the strain, and deformation potential are included into our three dimensional (3D) Poisson solver. The band structures are solved by $k \cdot p$ method. As we know, due to the effect of strong polarization field, a strong QCSE exists in nitride system, which decreases the emission strength. The band bending will decrease as the carrier injection density increases due to the 
screening effect. Therefore, in order to accurately estimate the band structures, a self-consistent method is necessary for high carrier density condition. However, in most of quantum dot simulation, ${ }^{9,12-16}$ these equations are not solved by a selfconsistent way, which is only valid for low carrier injection condition. In order to more accurately calculate the device, we have applied the self-consistent method to solve these equations in order to provide better estimation for high carrier injection condition. In this paper, we will systematically study the electronic and optoelectronic characteristics of InGaN/InN quantum dot structures by considering the effects of different dot sizes, indium compositions, wetting layer thickness, shapes, and carrier injection and compare them to those of quantum well structures. With these information, we can provide the experimentalists a guide of designing quantum dot devices. We think this will be very useful not only for lighting applications but also quantum dot laser applications.

\section{FORMALISM}

To understand the quantum dot system we need to address the following issues: (1) what is the strain tensor in the dots? (2) what is the polarization field in the dots? (3) what are the electronic states? and (4) what are the optical properties? Finally we need to compare the dot based results to the well based results. To study the strain tensor in InGaN quantum dot devices, we apply our previously developed simulation program based on the VFF model. ${ }^{18}$ The VFF model is a microscopic model where the interaction between each atom and its nearby atoms is considered. The total strain energy is expressed as function of atomic positions, $\vec{R}_{i}$, using a sum of bond stretching $\left(V_{2}\right)$ and bond bending $\left(V_{3}\right)$ terms:

$$
\begin{aligned}
E_{A E}= & \sum_{i j} V_{2}\left(\vec{R}_{i}-\vec{R}_{j}\right)+\sum_{i j k} V_{3}\left(\hat{\theta}_{i j k}\right)=\frac{1}{2} \sum_{i} \sum_{j}^{n n} \frac{3 \alpha_{i j k}}{8\left(d_{i j}^{0}\right)^{2}}\left[\left(\vec{R}_{i}\right.\right. \\
& \left.\left.-\vec{R}_{j}\right)^{2}-\left(d_{i j}^{0}\right)^{2}\right]^{2}+\frac{1}{2} \sum_{i} \sum_{j, k>j}^{n n} \frac{3 \beta_{i j k}}{8 d_{i j}^{0} d_{i j}^{0}}\left[( \vec { R } _ { j } - \vec { R } _ { i } ) \cdot \left(\vec{R}_{k}\right.\right. \\
& \left.\left.-\vec{R}_{i}\right)-\cos \theta_{0} d_{i j}^{0} d_{i j}^{0}\right]^{2},
\end{aligned}
$$

where $d_{i j}^{0}$ denotes the unstrained bond length between atoms $i$ and $j$, and $\theta_{0}$ is the unstrained bond angle, and $\cos \theta_{0}=$ $-1 / 3$. The bond stretching $\alpha$ and bond bending $\beta$ force constants are listed in Mattila and Zunger. ${ }^{18}$ The strain energy of each atom is minimized by an iterative method to find the most stable configuration. Once the system reaches the lowest energy, the strain distribution inside the structure can be obtained by evaluating the relative shift of each atom in a cell. The strain is obtained in the dots as well as the surrounding $\mathrm{GaN}$ region as well as in any wetting layer present.

After obtaining the strain information, we calculate the induced piezoelectric polar charge in the quantum dot device. The polarization induced by strain can be obtained by

$$
\begin{aligned}
& P_{e z}=e_{31}\left(\epsilon_{x x}+\epsilon_{y y}\right)+e_{33} \epsilon_{z z}, \\
& P_{e x}=e_{51} \epsilon_{z x},
\end{aligned}
$$

$$
P_{e y}=e_{51} \epsilon_{y z},
$$

where $e_{51}, e_{31}$, and $e_{33}$ are the piezoelectric coefficients and can be found in Ref. 21. After obtaining the piezoelectric polarization from strain and also considering the spontaneous polarization, we can calculate the total charge density from the total polarization. The potential affected by these polarization charges is solved with a 3D Poisson solver where

$$
\nabla^{2} V(x, y, z)=-\frac{\rho_{p}}{\varepsilon} .
$$

$\rho_{p}$ represents to total polarization charges, $V$ is the potential of the quantum dot device, and $\varepsilon$ is the dielectric constant. The LAPACK solver is applied to solve the large sparse matrix, where the band size of the sparse matrix is minimized and banded matrix solver in LAPACK is used to get the inverse. Finally, in order to calculate the eigenstates and envelope wave function of electrons and holes, we applied 6 $\times 6 k \cdot p$ envelope function method ${ }^{22,23}$ for calculating the valence band and effective mass approximation method for calculating the conduction band. The $6 \times 6 k \cdot p$ envelope function model can be expressed as

$$
H^{v}=\left[\begin{array}{llllll}
F & 0 & -H^{*} & 0 & K^{*} & 0 \\
0 & G & \Delta & -H^{*} & 0 & K^{*} \\
-H & \Delta & \lambda & 0 & I^{*} & 0 \\
0 & -H & 0 & \lambda & \Delta & I^{*} \\
K & 0 & I & \Delta & G & 0 \\
0 & K & 0 & I & 0 & F
\end{array}\right]\left[\begin{array}{l}
\Phi_{1}^{v} \\
\Phi_{2}^{v} \\
\Phi_{3}^{v} \\
\Phi_{4}^{v} \\
\Phi_{5}^{v} \\
\Phi_{6}^{v}
\end{array}\right],
$$

where

$$
\begin{aligned}
& F=\Delta_{1}+\Delta_{2}+\lambda+\theta, \\
& G=\Delta_{1}-\Delta_{2}+\lambda+\theta, \\
& H=i\left(A_{6} k_{z} k_{+}+A_{7} k_{+}+D_{6} \epsilon_{z+}\right), \\
& I=i\left(A_{6} k_{z} k_{+}-A_{7} k_{+}+D_{6} \epsilon_{z+}\right), \\
& K=A_{5} k_{+}^{2}+D_{5} \epsilon_{+}, \quad \Delta=\sqrt{2} \Delta_{3}, \\
& \lambda=A_{1} k_{z}^{2}+A_{2} k_{\perp}^{2}+D_{1} \epsilon_{z z}+D_{2}\left(\epsilon_{x x}+\epsilon_{y y}\right), \\
& \theta=A_{3} k_{z}^{2}+A_{4} k_{\perp}^{2}+D_{3} \epsilon_{z z}+D_{4}\left(\epsilon_{x x}+\epsilon_{y y}\right), \\
& \epsilon_{+}=\epsilon_{x x}-\epsilon_{y y}+2 i \epsilon_{x y}, \\
& \epsilon_{z+}=\epsilon_{x z}+i \epsilon_{y z}, \\
& k_{+}=k_{x}+i k_{y}, \quad \text { and } \\
& k_{\perp}^{2}=k_{x}^{2}+k_{y}^{2} .
\end{aligned}
$$

$D_{1}-D_{6}$ are the deformation potentials and $A_{1}-A_{7}$ are the fitting parameters to valence band structures, which can be found in Ref. 24. The $k_{x}, k_{y}$, and $k_{z}$ will be transformed into the differential forms $-i \partial / \partial x,-i \partial / \partial y$, and $-i \partial / \partial z$, respectively. The bases of the Hamiltonian are $1 / \sqrt{2}|X+i Y, \uparrow\rangle$, 
$1 / \sqrt{2}|X+i Y, \downarrow\rangle,|Z, \uparrow\rangle,|Z, \downarrow\rangle, 1 / \sqrt{2}|X-i Y, \uparrow\rangle$, and $1 / \sqrt{2} \mid X$ $-i Y, \downarrow\rangle$, where the polarization of the emission light is strongly affected by these basis functions. A 3D finite difference method is applied to solve the $6 \times 6 k \cdot p$ method, where the matrix size is $6 N^{3}$ and $N$ is the discretization segment. The ARPACK solver is used to find the eigenvalue and wave function for this large sparse matrix. In order to compare with the quantum well system, a self-consistent PoissonSchrödinger solver developed by Wu et $a l^{25}$ is used to study the band structure of quantum well for comparison. The $k \cdot p$ method is applied in the quantum well structure to calculate the electron and hole wave functions near the band edge.

After obtaining the e-h wave function and energy levels, we can calculate the spontaneous emission rates of a single quantum dot. The light emission rates is expressed as

$$
\begin{aligned}
R_{\text {spon }}= & \int d(\hbar \omega) \frac{2 e^{2} n_{r} \hbar \omega}{m_{0}^{2} \varepsilon_{0} c^{3} \hbar^{2}} \sum_{i, j}\left|\hat{a} \cdot \mathbf{p}_{\mathbf{i}, \mathbf{j}}\right|^{2} \\
& \times \frac{1}{\sqrt{2 \pi} \sigma} \exp \left(\frac{-\left(E_{i, j}-\hbar \omega\right)^{2}}{2 \sigma^{2}}\right) f^{e}\left(E_{i}^{e}\right) f^{h}\left(E_{j}^{h}\right), \quad\left(\mathrm{s}^{-1}\right),
\end{aligned}
$$

where $E_{i, j}$ is the average energy separation between $i, j$ states, $n_{r}$ is the refractive index, and $\sigma$ is the inhomogeneous broadening factor. $f^{e}$ and $f^{h}$ are the occupancy of electrons and holes, which can be expressed as

$$
\begin{aligned}
f^{e}\left(E_{i}^{e}\right) & =\frac{1}{1+\exp \left[\left(E_{i}-E_{f n}\right) / k_{B} T\right]}, \\
f^{h}\left(E_{j}^{h}\right) & =\frac{1}{1+\exp \left[\left(E_{f p}-E_{j}\right) / k_{B} T\right]} .
\end{aligned}
$$

$E_{f n}$ and $E_{f p}$ are the quasi-Fermi levels and will be decided by the injected carrier density. $\left|\hat{a} \cdot \mathbf{p}_{\mathbf{i}, \mathbf{j}}\right|^{2}$ is the momentum matrix element between electronic state $i$ and hole state $j$ and can be expressed as

$$
\left|\hat{a} \cdot \mathbf{p}_{\mathbf{i}, \mathbf{j}}\right|^{2}=\left|\left\langle u_{c}|\hat{a} \cdot \mathbf{p}| u_{v}\right\rangle\right|^{2}\left|\left\langle\Phi_{i}^{c} \mid \Phi_{j}^{v}\right\rangle\right|^{2}
$$

The emission strength will be basically affected by the square of the electron-hole (e-h) envelope function overlap, $\left|\left\langle\Phi_{i}^{c} \mid \Phi_{j}^{v}\right\rangle\right|^{2}$, which will be discussed in great detail in Sec. III.

The carrier radiative lifetime can be calculated by

$$
\tau_{r}=\frac{1}{n_{\mathrm{inj}} R_{\mathrm{spon}}}(\mathrm{s}),
$$

where $n_{\text {inj }}$ is the injected carrier numbers. With these formalisms, we can study the performance of the quantum dot device.

\section{A. A self-consistent solver for solving Poisson and $k \cdot \boldsymbol{p}$ Schrödinger equation}

As mentioned earlier, when carrier are injected into the InGaN quantum dot device, the QCSE will be screened by the free carriers. As a result, the band bending will decrease, the oscillator strength will increase, and the emission peak will have a blue shift. Self-consistent approaches have been applied to quantum well system where it is shown that for

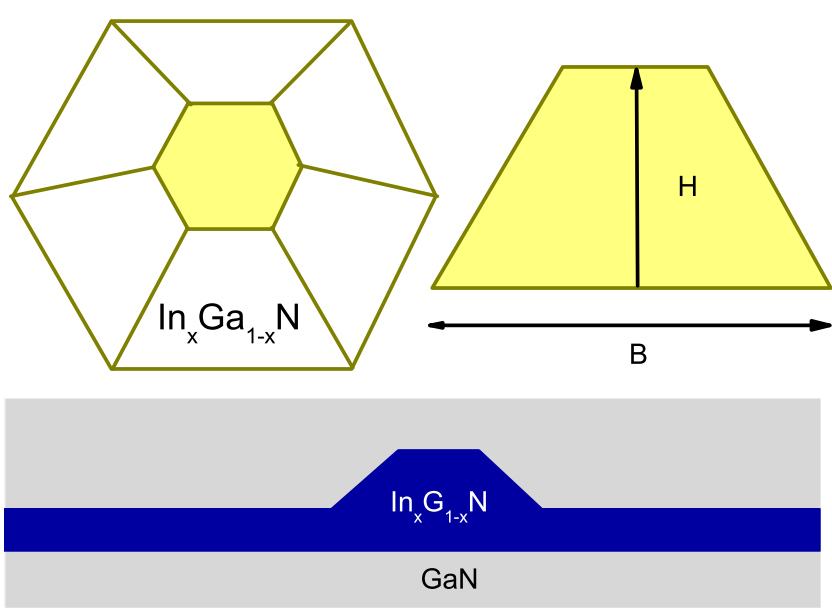

FIG. 1. (Color online) A schematic of quantum dot structure examined in this paper.

$c$-axis growth, there are strong screening effects that cannot be ignored. Large blueshift (up to $100 \mathrm{meV}$ ) and strong change in oscillator strength (up to a factor of 3) occur when one goes to high injection. For quantum dots, such selfconsistent approaches are not available. This is mostly due to the enormous numerical challenges affected by 3D system of equations that need to be solved. Thus the question remains if whether self-consistent studies are critical in understanding quantum dots, especially at high injection levels needed for lighting or laser applications. In this paper, we present selfconsistent solutions for the electronic and optical properties of InGaN quantum dots. Due to the computational complexities, the results are presented for a few selected cases under high injection conditions.

\section{RESULTS}

Figure 1 shows a schematic of the quantum dot structure we consider in the calculation. Since the InGaN is a wurtzite structure with hexagonal symmetry. Therefore, the hexagonal pyramid is mainly considered in this paper. ${ }^{12,14}$ Some work $^{9}$ suggested that different dot shapes will affect the emission property, which is reasonable due to different strain effects. However, for most experimental works, the dot shape is more close to hexagonal pyramid so that we mainly focus on this shape. The base to height $(\mathrm{BH})$ ratio in the calculation ranges from about $2-4$. Note that the ratio is not exactly 2 or 4 since the lattice dimensions of $a$ and $c$ are not the same. It is known that quantum dots are formed on top of a wetting layer. We calculate results with wetting layer thicknesses ranging from $\sim 0$ to $1.08 \mathrm{~nm}$. For the thicker wetting layer, the behavior is found to be more like that in a quantum well where no localized confined state is found and very strong piezoelectric effect is induced inside the wetting layer.

The strain inside the quantum dot not only influences the deformation potential (and thus bandgap) but also the piezoelectric polarization. Figure 2 shows the strain of $\operatorname{In}_{0.2} \mathrm{Ga}_{0.8} \mathrm{~N}$ quantum dot with height of $4.3 \mathrm{~nm}$. The wetting layer thickness is $0.53 \mathrm{~nm}$ and the $\mathrm{BH}$ value is equal to 2. Figures 2(a) and 2(b) are the cross section view of $\epsilon_{x x}$ and $\epsilon_{z z}$ at the center of the $y$-axis, respectively. Figures 2(c) and 2(d) are the top view of $\epsilon_{x x}$ and $\epsilon_{z z}$ at $z=4 \mathrm{~nm}$, respectively. As shown in 

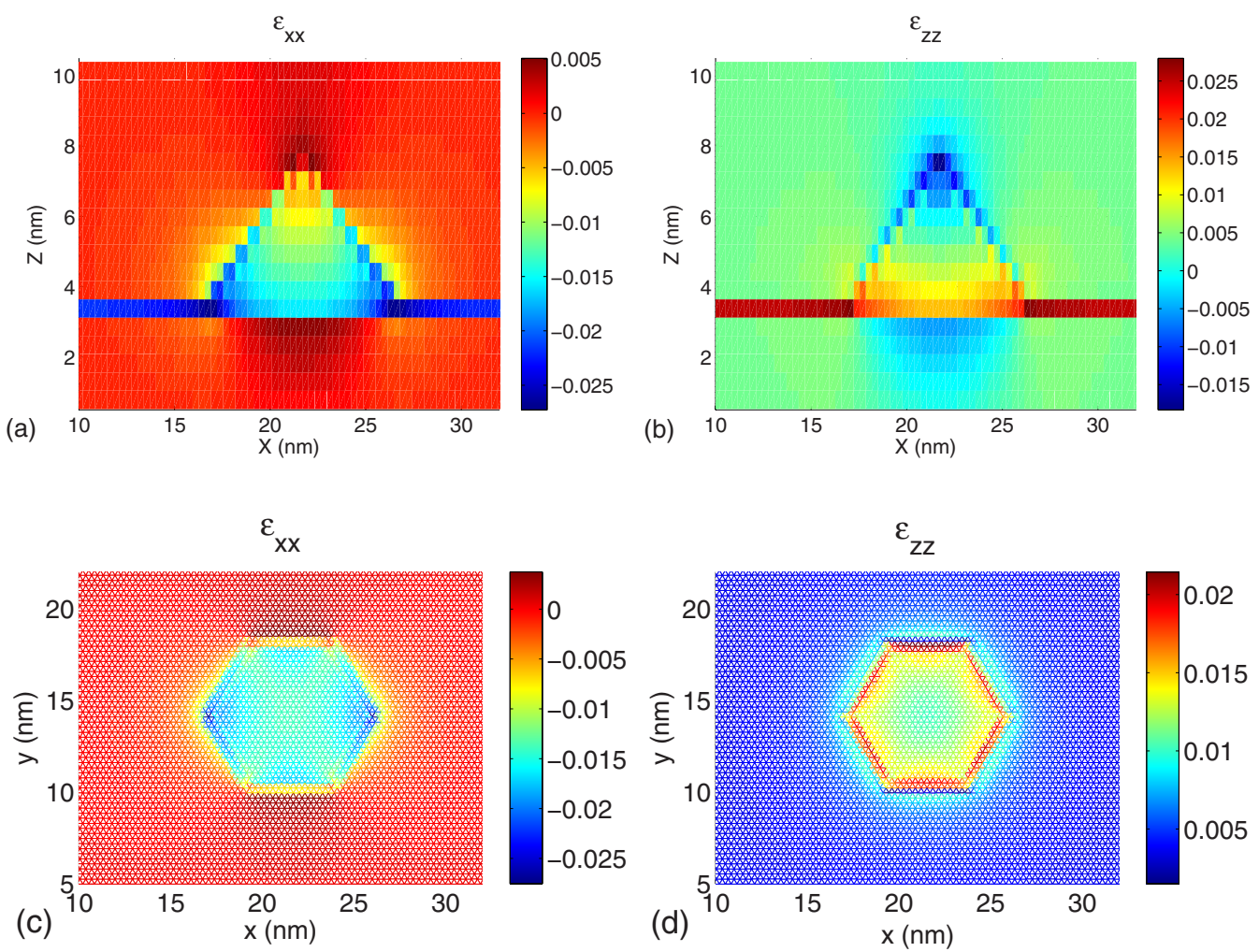

FIG. 2. (Color online) (a) and (b) are the cross section view of $\epsilon_{x x}$ and $\epsilon_{z z}$ at center of the $y$ axis, respectively. (c) and (d) are the top view of $\epsilon_{x x}$ and $\epsilon_{z z}$ at $z=4 \mathrm{~nm}$, respectively. The dot height is $4.3 \mathrm{~nm}$, the wetting layer thickness is $0.53 \mathrm{~nm}$ and the $\mathrm{BH}$ value is 2 .

Fig. 2(a), there is a strong compressive strain, $\boldsymbol{\epsilon}_{x x}$, in the InGaN wetting layer at bottom and the compressive strain becomes slightly weaker toward the top of the InGaN quantum dot, and as a result the $\mathrm{GaN}$ region right on the top corner of the dot will suffer a slight tensile strain. Additionally there is a very strong tensile strain $\epsilon_{z z}$ at bottom of InGaN and a slightly compressive strain at the top of the InGaN dot as shown in Fig. 2(b). The strain induced polarization charges can be calculated by Eq. (2). We find that the strain variation is much smaller in the dot than the strain in the wetting layer. Therefore, the polarization charge distribution is much smoother and the potential variation is much weaker.

Figure 3 shows the calculated piezoelectric polarization charge distribution inside the quantum dot for the height equal to $4.3 \mathrm{~nm}$. As shown in Fig. 3, the polarization charge density is much weaker compared to the wetting layer since the strain is relaxed in the dot structures. The potential variation due to the polarization charges is calculated by 3D Poisson solver as described in Eq. (5) and Fig. 4 is one of these results. The results show that there is $\sim 0.25 \mathrm{eV}$ band bending inside the quantum dot device, which is much weaker than in typical quantum well cases for the same thickness. This would lead to a stronger e-h overlap. However, it is also observed that the polarization charges induced by the wetting layer will contribute to the additional band bending and as a result, a thinner wetting layer or dots without a wetting layer would be ideal choices to optimize the device performance. Note that the result is obtained without carrier injection. The band bending will be weaker after carriers are injected.

For ideal white light emitting sources, one would like to have light emitters that can emit in the entire visible light spectrum. Figure 5 shows the change in effective bandgap versus dot height with different indium compositions. For optimization, no wetting layer is set and the BHs of Figs. 5(a) and 5(b) are 2 and 4, respectively. As the dot size increases, it is expected that the effective bandgap will decrease. As the $\mathrm{BH}$ values become larger, the device behaves more likely to the quantum well structure, where the quantum confinement effect is weaker and the effective bandgap is smaller compared the same dot height with smaller $\mathrm{BH}$. The results suggest that we can design quantum dots to emit light with a wide spectral range by controlling the dot size and indium composition to make a more saturated or tunable

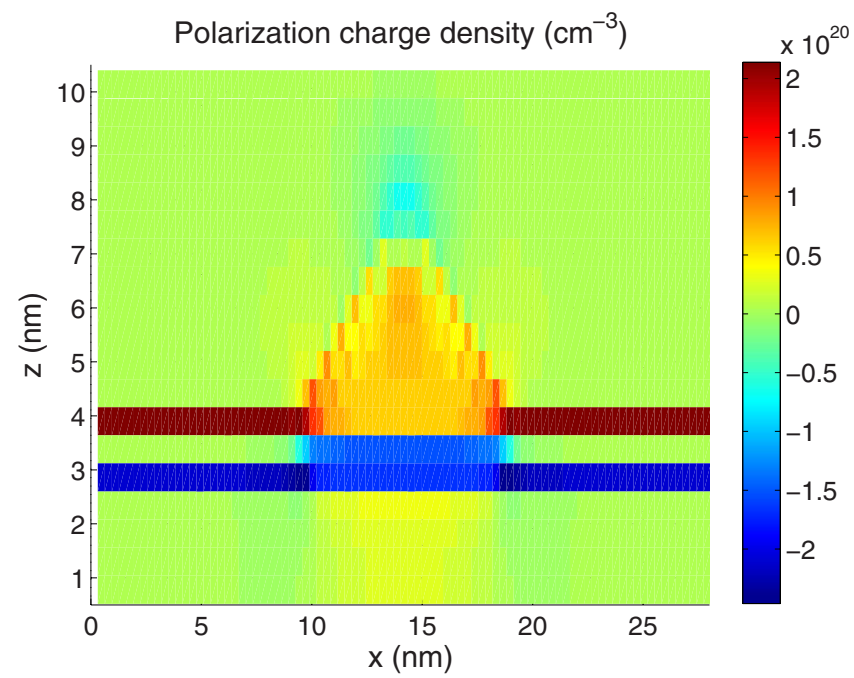

FIG. 3. (Color online) The side view of the calculated piezoelectric polarization charge density in the quantum dot device. 


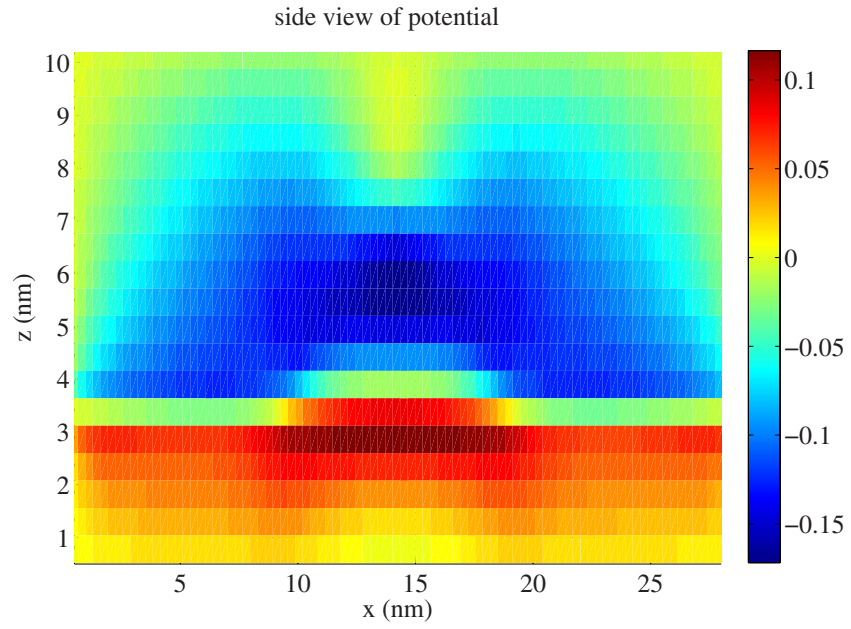

FIG. 4. (Color online) The potential variation in the quantum dot device due to the piezoelectric polarization.

white light sources thus achieving performance better than the current phosphor coating based devices. It is note that when the dot size is too small, we might not be able to find the confined electronic states. Therefore, for small indium composition cases such as $\operatorname{In}_{0.2} \mathrm{Ga}_{0.8} \mathrm{~N}$ quantum dots, where the barrier potential is small, the shrinkage of dot size is further limited.

Figure 6 shows the overlap of e-h ground state wave functions and the calculated radiative lifetime of a single quantum dot. The radiative lifetime is calculated by assuming that one electron and one hole are injected into the quantum dot. The BH values of Figs. 6(a) and 6(b) are 2 and 4, respectively. No wetting layer is assumed in this case. As shown in Fig. 6, the e-h overlap reaches 50\%-70\% for dot sizes around 2-3 nm. As the dot size continues to decrease, the electron, which has smaller effective mass, will not be well confined inside the dot and the wave function will spread into barrier region so that the further reduction in dot size is limited. Also when the indium composition increases, the electron is better confined in the quantum dot so that a slightly stronger overlap is achieved at the smaller dot size. The overlap decreases gradually as the dot size increases due to the QCSE. As the BH values become larger, the overlap decreases more rapidly as the indium composition increases.

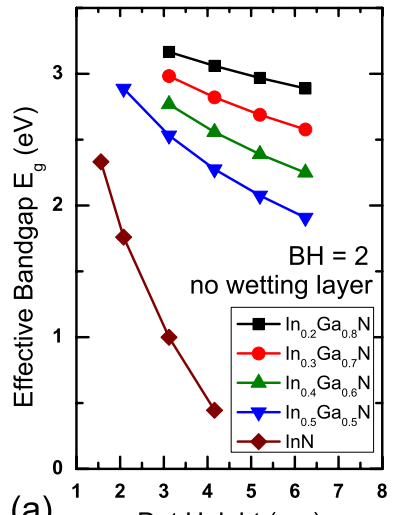

(a) Dot Height (nm)

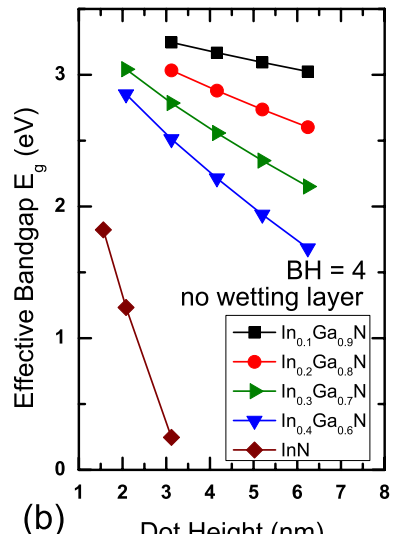

FIG. 5. (Color online) The calculated effective bandgap in the quantum dot device with different indium concentrations and dot sizes; (a) is the case for $\mathrm{BH}=2$ and $(\mathrm{b})$ is for $\mathrm{BH}=4$.
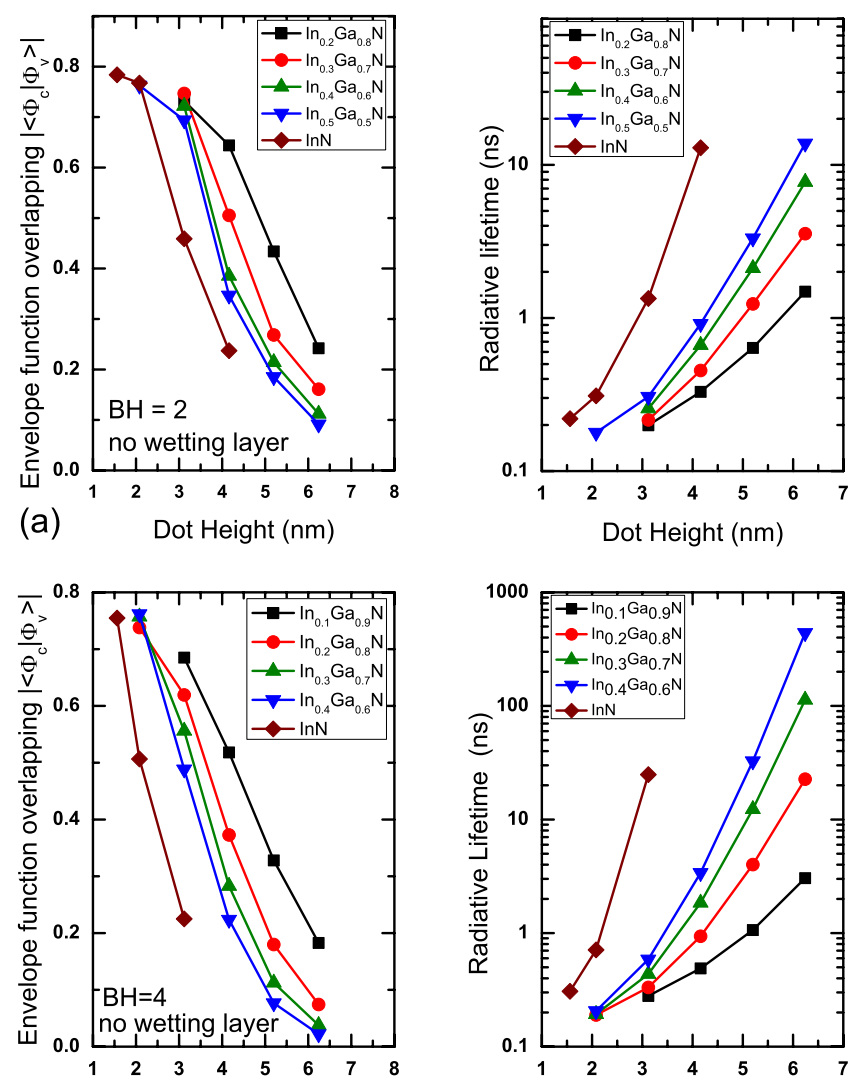

(b)

Dot Height (nm)

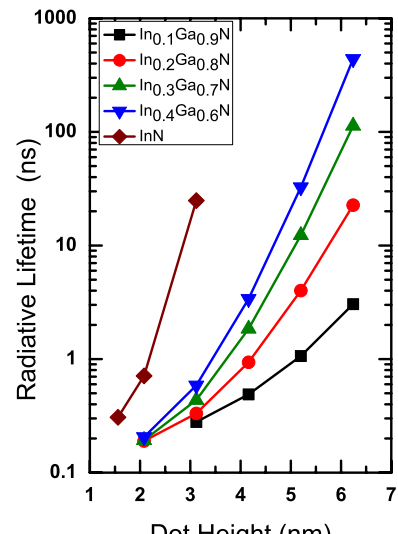

FIG. 6. (Color online) The e-h overlap and calculated lifetime in the quantum dot device with different dot sizes and indium concentrations. The radiative lifetime is calculated by assuming one electron and hole injected into the quantum well. (a) and (b) are the cases for $\mathrm{BH}=2$ and $\mathrm{BH}=4$, respectively.

This may be due to the weaker confinement of quantum dot structures, where larger $\mathrm{BH}$ values with the same height have larger volume and thus reduce the quantum confinement effect. As the base to height ratio continues to increase, the device behavior will become more similar to the quantum well structure. The radiative lifetimes are calculated by Eqs. (7) and (10). From these equations, we can know that the radiative lifetime is mainly affected by the square of e-h overlap and injected carrier density. As shown in Fig. 6, the radiative lifetimes for $\mathrm{BH}$ equal to 2 are around $0.2-10 \mathrm{~ns}$ and the radiative lifetimes for $\mathrm{BH}$ equal to 4 are around $0.3-400 \mathrm{~ns}$. The results suggest that the dot size in the lateral direction is also limited.

As mentioned earlier, the wetting layer thickness will affect the e-h overlap significantly. As we know, for an InGaN quantum well, the typical well thickness is around 2-3 $\mathrm{nm}$. Therefore, if the wetting layer is too large, the strong polarization between the interfaces will introduce an extra band bending to reduce the oscillator strength. As shown in Fig. 7, a slightly increase in the wetting layer reduces the e-h overlap. The emission rate will drop since it is proportional to the square of e-h overlap. The results suggest that a quantum dot without a wetting layer could provide a better performance. Some growth technologies such as directly using E-beam to make a nanoscale mask for the nanodot growth might be able to make structures without a wetting layer.

In order to examine the performance of quantum dot as 


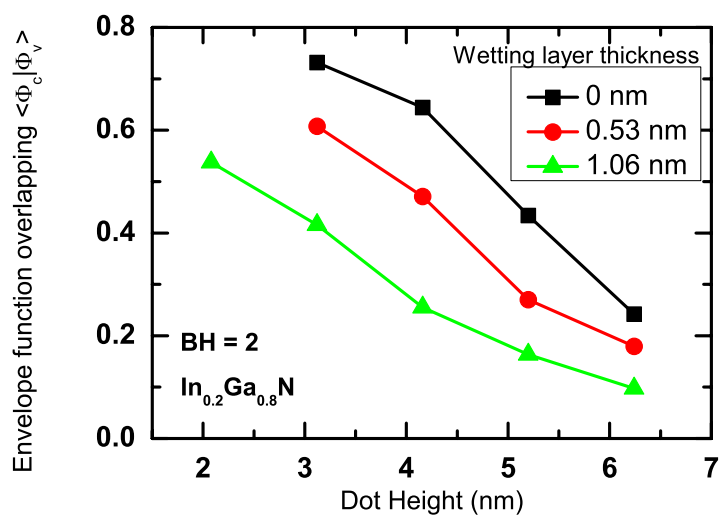

FIG. 7. (Color online) The e-h overlap in the $\operatorname{In}_{0.2} \mathrm{Ga}_{0.8} \mathrm{~N}$ quantum dot device vs different wetting layer thicknesses. The $\mathrm{BH}$ ratio is 2 .

compared to the quantum well case, we also calculate the oscillator strength and effective bandgap for quantum well structures with different well sizes. Figure 8(a) shows the calculation results for $\operatorname{In}_{0.2} \mathrm{Ga}_{0.8} \mathrm{~N}$ quantum wells with different well sizes and different injection conditions. As shown in Fig. 8(a), the overlap of e-h envelope function of quantum well structures is only about $39 \%$ at low injection conditions and about $53 \%$ under high injection condition for $2 \mathrm{~nm}$ well width. Additionally the e-h overlap drops rapidly when the well thickness increases. Therefore, the quantum dot structure could have a $60 \%-70 \%$ improvement in e-h overlaps
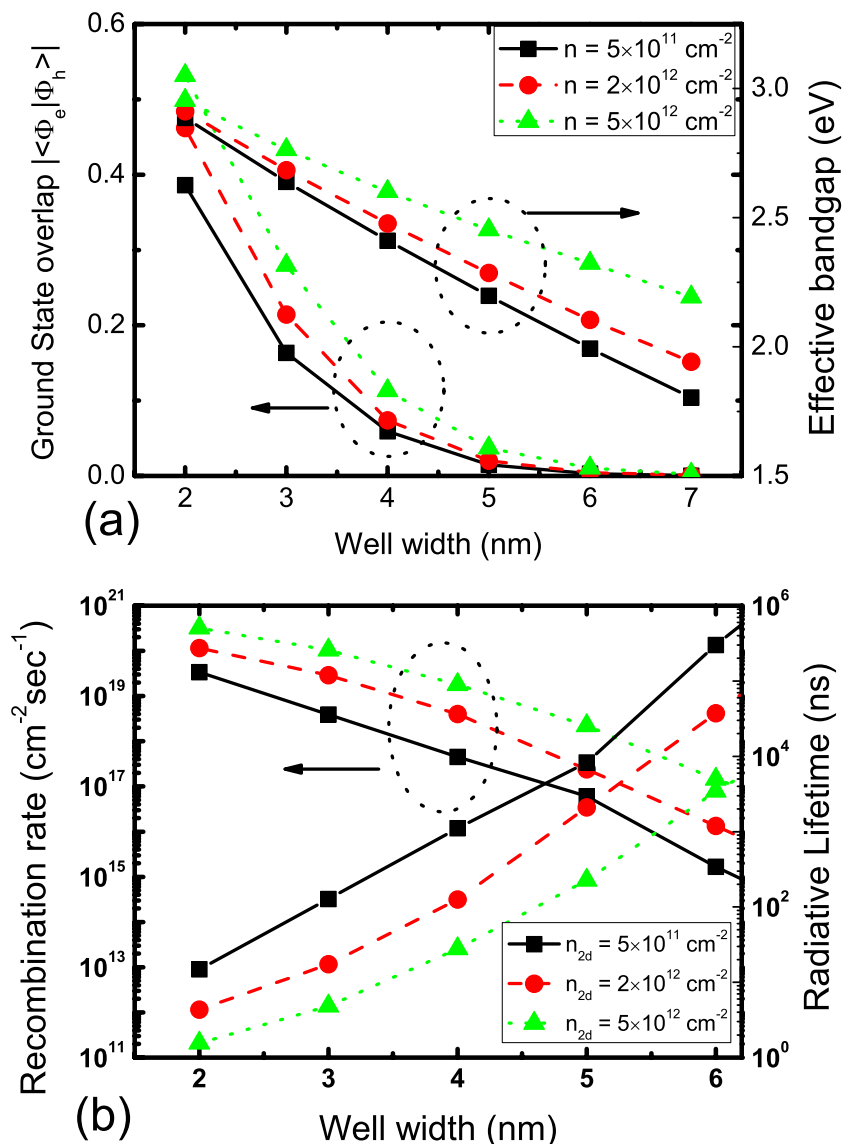

FIG. 8. (Color online) (a) The calculated e-h overlap and effective bandgap in the quantum well device with different injection carrier densities. (b) The calculated radiative recombination rates and radiative lifetimes of the quantum well with different injection carrier densities. compared to the single quantum well cases. For smaller well width cases, the injected carrier density is limited so that the carriers cannot fully screen the polarization field.

Figure 8(b) shows the calculated radiative lifetime and the corresponding spontaneous emission rates for the quantum well case with different quantum well thicknesses. As shown in the figure, a higher e-h overlap will lead to shorter radiative lifetime, which can greatly improve the internal quantum efficiency in the light emitter in presence of defects. When the quantum well thickness is larger than $4 \mathrm{~nm}$, the radiative lifetime is much larger than $100 \mathrm{~ns}$, which is too large for light emission.

The radiative lifetimes for quantum well structures are much larger than what we calculate for the quantum dot structures where typical radiative lifetimes are found to be around $0.3 \mathrm{~ns}$ to a few nanoseconds. It is also important to note that in the quantum well structures, although the electron and hole wave functions are confined in the vertical direction, they are free to move in the lateral direction. This could increase the chance of nonradiative lifetime due to defects, dislocations, etc., since carriers could diffuse to these defects. In the quantum dot device, the electron and hole are confined inside the dot state and since in StranskiKrastanov growth there is no dislocation through the quantum dot the nonradiative lifetime could be longer, which could improve the internal quantum efficiency even more.

If we assume that the quantum dot density is around 5 $\times 10^{11} \mathrm{~cm}^{-2}$ in a layer and each dot is injected by one electron and hole, which is equal to the quantum well case where $n_{2 \mathrm{D}}=5 \times 10^{11} \mathrm{~cm}^{-2}$, we can find that the radiative lifetime of quantum dot is two order of magnitude smaller than the quantum well cases. This implies larger current can be injected into a quantum dot layer compared to single quantum well cases. Even if the quantum dot density is around $10^{10} \mathrm{~cm}^{-2}$, the quantum dot device is still competitive to the quantum well structures. It may also be possible to stack quantum dots to increase their areal density.

As mentioned earlier, it is important to understand how the injected carriers screen the electric polarization field. Therefore, we applied the self-consistent approach to study changes in the oscillator strength when carriers are injected. Figure 9(a) shows the potential along the $z$ axis at the center of the quantum dot device with dot height equal to $4.3 \mathrm{~nm}$. The wetting layer thickness is $0.53 \mathrm{~nm}$. As shown in Fig. 9(a), the band bending is stronger when carriers are not injected. As the electron and hole are injected, we can find the relative changes to the potential. When we injected more than two electron and hole into a single quantum dot, the screening effect becomes saturated since higher order state is not well localized inside the quantum dot. Figure 9(b) shows the changes in the effective bandgap. A maximum blueshift around $10-50 \mathrm{meV}$ is observed in high injection condition. Figure 9(c) shows the changes in the e-h overlaps and estimated radiative lifetimes versus injected carrier numbers. There is $10 \%-30 \%$ changes in e-h overlap when the electron and hole are injected. The screening effect is much clear for larger dot size due to the larger band bending in the system. The radiative lifetime is inversely proportional the square of e-h overlap so that a $70 \%$ improvement is expected for the 

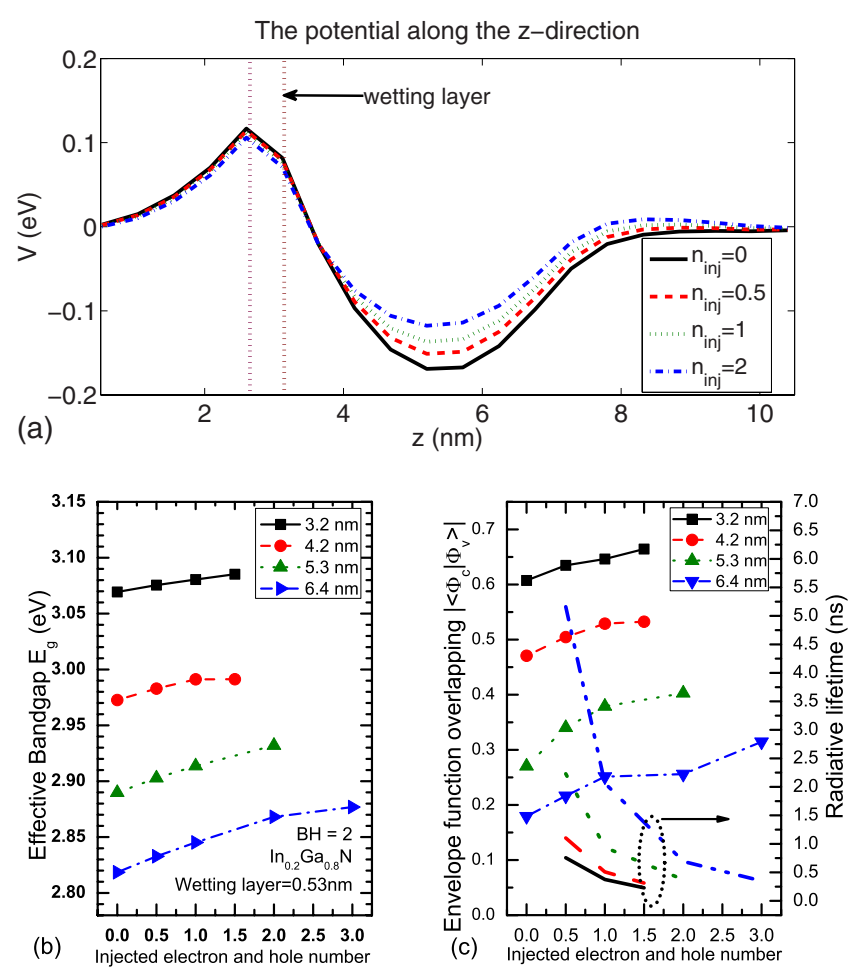

FIG. 9. (Color online) (a) shows the calculated potential inside the quantum dot for different injected carrier numbers by the self-consistent method, which includes the carrier screening effects of the polarization field; (b) shows the shift of the effective bandgap with different carrier injections. (c) The calculated lifetime and e-h overlap vs injected carrier number for different dot sizes.

dot size equal to $6.4 \mathrm{~nm}$. Continues to increase the carrier injection may increase the e-h overlap. However, it will be limited by the dot size due to the quantum confined effect. Only a few carriers can be injected in the quantum dot device especially for the small one. Our result suggests that under very high carrier injection condition, the self-consistent method will be needed for better consideration of device performance.

In this paper, we have studied the performance of InGaN quantum dot with different dot sizes, shapes, indium compositions, and wetting layer thicknesses. If the StranskiKrastanov method is used to grow the InGaN quantum dot, the wetting layer is expected to be around 1-2 $\mathrm{nm}$. In our study, we find that this will affect the e-h overlap. However, since the electron and hole are in a localized state, they are not moving laterally and may thus be unaffected by dislocations or defects. Therefore, even if the radiative lifetime is long, the nonradiative lifetime could be even longer.

\section{CONCLUSION}

In conclusion, we have made a systematic study of the band structure of InGaN quantum dot devices and compared the results with InGaN quantum well devices. Our results suggest that quantum dot based devices could provide better e-h overlap to reduce the radiative lifetime. The effects of wetting layer, dot size, indium composition, and $\mathrm{BH}$ ratio have been evaluated and it suggests that a thinner wetting layer and smaller base to height ratios are suggested for op- timum devices. Of course, this depends on fabrication ability. It also depends on the barrier potential to confine the electrons. The relation of dot size versus the effective bandgap for different indium compositions is also studied. Our calculated results provide a guideline for controlling emission wavelength of quantum dots with different dot sizes and indium compositions. The oscillator strength of quantum dot is much larger than the quantum well devices. We have also studied the band bending and oscillator strength change when injection densities are high. A self-consistent method has been applied to study the screening of electric polarization. We see that while screening effects are not as strong as for quantum wells there are still some improvements in e-h overlap and carrier recombination. The result suggests that typically recombination rates can be enhanced by more than $70 \%$ when the structure has a high level of carrier injection.

\section{ACKNOWLEDGMENTS}

This work was supported by the National Science Council in Taiwan under Grant No. 96-2218-E-002-031, 97-2221E-002-050, and by the DOE under Contract No. DEFC2607NT43229. Brian Dotson is the contract monitor of DOE.

${ }^{1}$ Y. Narukawa, J. Narita, T. Sakamoto, K. Deguchi, T. Yamada, and T. Mukai, Jpn. J. Appl. Phys., Part 2 45, L1084 (2006).

${ }^{2}$ H. W. Huang, C. F. Lai, W. C. Wang, T. C. Lu, H. C. Kuo, S. C. Wang, R. J. Tsai, and C. C. Yu, Electrochem. Solid-State Lett. 10, H59 (2007).

${ }^{3}$ Y.-L. Li, Y.-R. Huang, and Y.-H. Lai, Appl. Phys. Lett. 91, 181113 (2007).

${ }^{4}$ M.-H. Kim, M. F. Schubert, Q. Dai, J. K. Kim, E. F. Schubert, J. Piprek, and Y. Park, Appl. Phys. Lett. 91, 183507 (2007).

${ }^{5}$ K. Tachibana, T. Someya, and Y. Arakawa, Appl. Phys. Lett. 74, 383 (1999).

${ }^{6}$ R. A. Oliver, G. A. D. Briggs, M. J. Kappers, C. J. Humphreys, S. Yasin, J. H. Rice, J. D. Smith, and R. A. Taylor, Appl. Phys. Lett. 83, 755 (2003).

${ }^{7}$ D. Simeonov, E. Feltin, J.-F. Carlin, R. Butte, M. Ilegems, and N. Grandjean, J. Appl. Phys. 99, 083509 (2006).

${ }^{8}$ N. Grandjean and M. Ilegems, Proc. IEEE 95, 1853 (2007).

${ }^{9}$ M. Winkelnkemper, A. Schliwa, and D. Bimberg, Phys. Rev. B 74, 155322 (2006).

${ }^{10}$ M. Winkelnkemper, R. Seguin, S. Rodt, A. Schliwa, L. Reissmann, A. Strittmatter, A. Hoffmann, and D. Bimberg, Physica E 40, 2217 (2008).

${ }^{11}$ J. K. Park, K. J. Choi, H. G. Kang, J. M. Kim, and C. H. Kim, Electrochem. Solid-State Lett. 10, J15 (2007).

${ }^{12} \mathrm{~N}$. Vukmirović, Z. Gacevic, Z. Ikonic, D. Indjin, P. Harrison, and V. Milanovic, Semicond. Sci. Technol. 21, 1098 (2006).

${ }^{13}$ A. D. Andreev and E. P. O'Reilly, Phys. Rev. B 62, 15851 (2000).

${ }^{14}$ A. D. Andreev and E. P. O'Reilly, Appl. Phys. Lett. 79, 521 (2001).

${ }^{15}$ E. Sarigiannidou, E. Monroy, B. Daudin, J. L. Rouviere, and A. D. Andreev, Appl. Phys. Lett. 87, 203112 (2005).

${ }^{16}$ D. P. Williams, A. D. Andreev, and E. P. O'Reilly, Phys. Rev. B 73, 241301 (2006)

${ }^{17}$ P. Keating, Phys. Rev. 145, 637 (1966).

${ }^{18}$ T. Mattila and A. Zunger, J. Appl. Phys. 85, 160 (1999).

${ }^{19}$ H. Jiang and J. Singh, Physica E 2, 614 (1998).

${ }^{20} \mathrm{H}$. Jiang and J. Singh, Physica E 2, 720 (1998).

${ }^{21}$ O. Ambacher, J. Majewski, C. Miskys, A. Link, M. Hermann, M. Eickhoff, M. Stuzmann, F. Bernardini, V. Fiorentini, V. Tilak, B. Schaff, and L. F. Eastman, J. Phys.: Condens. Matter 14, 3399 (2002).

${ }^{22}$ M. Suzuki, T. Uenoyama, and A. Yanase, Phys. Rev. B 52, 8132 (1995).

${ }^{23}$ S. Ghosh, P. Waltereit, O. Brandt, H. T. Grahn, and K. J. Ploog, Phys. Rev. B 65, 075202 (2002).

${ }^{24}$ S.-H. Park, D. Ahn, and S.-L. Chuang, IEEE J. Quantum Electron. 43, 1175 (2007).

${ }^{25}$ Y.-R. Wu, M. Singh, and J. Singh, J. Appl. Phys. 94, 5826 (2003). 